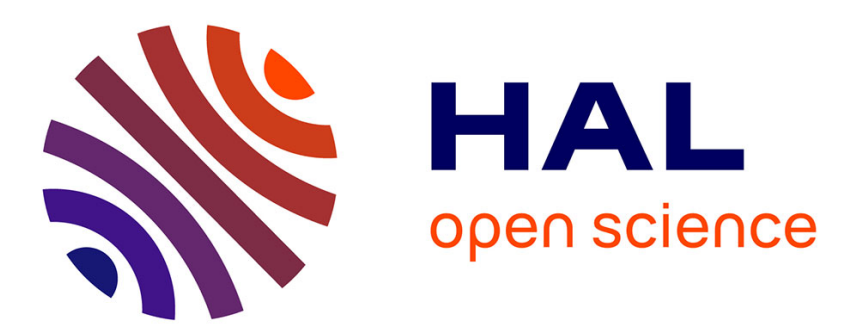

\title{
Escort function definitions for feasible region flexibility in applications of Replicator-like dynamics with constraints
}

\author{
Andres Ovalle, Ahmad Hably, Seddik Bacha
}

\section{- To cite this version:}

Andres Ovalle, Ahmad Hably, Seddik Bacha. Escort function definitions for feasible region flexibility in applications of Replicator-like dynamics with constraints. IEEE Transactions on Automatic Control, 2021, 66 (11), pp.5414-5421. 10.1109/TAC.2021.3065010 . hal-03172932

\section{HAL Id: hal-03172932 \\ https://hal.science/hal-03172932}

Submitted on 18 Mar 2021

HAL is a multi-disciplinary open access archive for the deposit and dissemination of scientific research documents, whether they are published or not. The documents may come from teaching and research institutions in France or abroad, or from public or private research centers.
L'archive ouverte pluridisciplinaire HAL, est destinée au dépôt et à la diffusion de documents scientifiques de niveau recherche, publiés ou non, émanant des établissements d'enseignement et de recherche français ou étrangers, des laboratoires publics ou privés. 


\title{
Escort function definitions for feasible region flexibility in applications of Replicator-like dynamics with constraints
}

\author{
Andres Ovalle, Student Member, IEEE, Ahmad Hably, Member, IEEE, and Seddik Bacha, Member, IEEE,
}

\begin{abstract}
This paper proposes an extension to a family of evolutionary game dynamics called Escort Replicator Dynamics (ERD), defined in [1] based on information-geometric concepts. Specifically, the objective of this paper is to propose three types of convenient so-called escort functions and evaluate the stability of ERD under the use of these functions. Since it is proven that ERD is stable using the proposed definitions, ERD is able to confine trajectories of the state vector in regions defined by upper and lower boundaries which can be, but are not required to be, non-negative. From an engineering application perspective, these proposed escort functions are convenient since they allow to extend replicator-like dynamics applications to engineering problems with feasible regions more general than the standard simplex or the non-negative orthant. From a population dynamics perspective the proposed escort functions are useful to represent upper and lower limits on the share of a population that is allowed to play a given pure strategy of the underlying symmetric game.
\end{abstract}

\section{INTRODUCTION}

Evolutionary game theory (EGT) explains the quantitative and qualitative aspects of evolution in biology by using game theory tools [2]. It models interactions between portions of a population (population dynamics) which try to maximize their wellness. Wellness is considered as the payoff obtained for using a certain combination of strategies. Portions of the population with strategies giving higher than average payoff will expand quicker and the proportion of these portions will tend to grow.

The concept of evolutionary stable strategy has been introduced to explain the level of effectiveness of a strategy in animal populations [3]. It attempts to capture the idea of resistance to mutations of succesful strategies in static environments. In other words, a population in which most of its members play an evolutionary stable strategy is resistant to invasion by a small group of mutants who play an alternative mixed strategy [4]. On the other hand, the concept of state of a population represents the distribution of this population over the available strategies. In other words, a state gathers information of the amounts of individuals of the population using each of the available strategies or mixed versions of them. An Evolutionarily Stable State (ESS) is one of these possible distributions with an important feature: if the population is in an ESS, after disturbances its genetic composition is restored by natural selection [2].

Reference [5] has mentioned the advantages of using EGT in engineering applications and shows its usefulness in several engineering examples such as smart lighting and optimal economic

The authors are with Université de Grenoble Alpes, CNRS, Grenoble INP, G2Elab, Gipsa-lab, F-38000 Grenoble, France. Ahmad Hably is the corresponding author, ahmad.hably@grenoble-inp.fr

This work is supported by the aVEnir (accompagnons le Véhicule Electrique avec la nécessaire intelligence de la recharge) project. dispatch in micro-grids. A similar approach has been used for distributed optimization in [6], for wireless networks in [7], and for load management of electric vehicle fleets in [8], [9]. EGT is also used for automating the clustering of nodes and nominations of cluster heads, to achieve cluster stability in Vehicular Ad hoc Networks in [10], and to analyze advanced persistent threats against cloud storage in [11]. Recently in [12], EGT has been used to analyze strategic choice between two competing alternatives having their own business case in employing upward consumption flexibility to alleviate problems caused by wind generation and renewable energy support schemes.

In [1], the functional parameter called Escort used in Escort Replicator Dynamics (ERD) has been introduced to make a generalization of evolutionary game dynamics (including replicator dynamics, and projection dynamics). This escort parameter can be interpreted an incentive (or deterrent) to the rate of grow of a population share. In [13] the local stability of the ERD, with certain escort functions, is proved by using generalized information divergencies (like the Kullback-Leibler divergence) as local Lyapunov functions. The selection of the candidate Lyapunov function depends on the escort functional parameter used with the ERD. Provided that this escort functions are nondecreasing and strictly positive on the interval $(0,1)$, authors of [13] describe several examples. Recently, the ERD has been considered as special case of the Separable Riemannian game dynamics described in [14, Example 4.3].

The main contribution of this manuscript is described in the following paragraphs. The general objective of the approach in this paper is to drive initial states to a local ESS (from its vicinity) for the underlying symmetric game (assuming an ESS exists in the interior of a considered feasible region). From an engineering application perspective, the underlying game can be, but is not required to be, defined by the euclidean gradient of a given potential function. In that context, the objective of driving states towards a local ESS is identical to that of finding a feasible optimum of the defining potential function in the interior of the feasible region, defined by upper and lower constraints on each state variable. From an EGT perspective, the objective is to reach a local ESS for the underlying game, constraining the size of shares of the population that are allowed to play each of the pure strategies. For instance this could represent limited adoption capacities for a given pure strategy, or minimal quotas in a particular scenario.

The main novelty in this paper is the exploitation the escort functions definition in ERD to confine population states in manifolds different than the standard simplex or the nonnegative orthant. Thus, feasible regions can be defined by upper 
and lower constraints, or the intersection of both. In an EGT context, the proposed escort functions are required to be defined for non-negative constraints so that a population share is never negative. However, in an engineering scenario, upper and lower boundaries are not required to be non-negative in the definition of the proposed escort functions.

The paper is organized as follows. In section III an introduction to the Escort Replicator Dynamics (ERD) is given. Then, the proposed escort functions are presented in Section III and a motivation is provided for these definitions. A formal stability proof is given in Section IV] It is followed by an illustrative example in Section VI The paper ends with a discussion in Section VII

\section{ESCORT REPLICATOR DYNAMICS FOUNDATION}

Since this manuscript works with the definitions presented in [1] for ERD, this section serves as an introduction to some of those definitions. Let us consider a population of individuals playing a game. In this set-up, all the individuals in the population are indistinguishable from each other, except for the strategy they chose to play in the evolutionary game. In other words, individuals in the population play a symmetric game where they all share the same finite set of pure strategies.

The generalized ERD, defined in continuous time by

$$
\dot{x}_{k}=\phi_{k}\left(x_{k}\right)\left(f_{k}(\mathbf{x})-\bar{f}_{\phi}(\mathbf{x})\right),
$$

describes the evolution of the distribution of this population over the finite set of $K$ possible pure strategies, according to the payoff these provide [1]. Here, the distribution at a given instant is represented by the state vector $\mathbf{x}=\left[x_{1}, x_{2}, \cdots, x_{k}, \cdots, x_{K}\right]^{T}$ whose elements $x_{k}$ represent the proportion of individuals in the population choosing pure strategy $k$, with payoff $f_{k}(\mathbf{x})$. In eq. (1), $\phi_{k}\left(x_{k}\right)$ is the so-called escort function. On the other hand, $\bar{f}_{\phi}(\mathbf{x})$ represents a weighted average payoff defined by,

$$
\bar{f}_{\phi}(\mathbf{x})=\frac{1}{\Phi(\mathbf{x})} \sum_{k=1}^{K} \phi_{k}\left(x_{k}\right) f_{k}(\mathbf{x}),
$$

where $\Phi(\mathbf{x})=\sum_{k=1}^{K} \phi_{k}\left(x_{k}\right)$. It can be noticed that if $\phi_{k}\left(x_{k}\right)=$ 0 at $t=0$, then $x_{k}$ will remain constant for $t>0$. Moreover, proportions $x_{k}$ with payoffs greater that the weighted average payoff (i.e. $f_{k}(\mathbf{x})>\bar{f}_{\phi}(\mathbf{x})$ ) will tend to grow and vice-versa.

The weighted average payoff defined by eq. (2) can be understood as the expected value of the vector of payoff functions given a probability distribution $\theta(\mathbf{x})$ defined by the escort functions as,

$$
\theta(\mathbf{x})=\frac{1}{\Phi(\mathbf{x})} \phi(\mathbf{x})=\frac{1}{\Phi(\mathbf{x})}\left[\phi_{1}\left(x_{1}\right), \cdots, \phi_{K}\left(x_{K}\right)\right]^{T} .
$$

On the other hand, let us sum eq. (1) over all the pure strategies played by individuals of the population,

$$
\begin{aligned}
\sum_{k=1}^{K} \dot{x}_{k} & =\sum_{k=1}^{K} \phi_{k}\left(x_{k}\right) f_{k}(\mathbf{x})-\bar{f}_{\phi}(\mathbf{x}) \sum_{k=1}^{K} \phi_{k}\left(x_{k}\right) \\
& =\Phi(\mathbf{x}) \bar{f}_{\phi}(\mathbf{x})-\Phi(\mathbf{x}) \bar{f}_{\phi}(\mathbf{x})=0 .
\end{aligned}
$$

Therefore, if at $t=0$ the state vector $\mathbf{x}$ lies in the hyper-plane defined by,

$$
\mathbb{H}_{m}^{K}=\left\{\mathbf{x} \in \mathbb{R}^{K}: \sum_{k=1}^{K} x_{k}=m\right\},
$$

then the state vector will remain in that hyper-plane for $t>0$, regardless of the escort functions that are consistently defined. Note that in the context of population dynamics, $m=1$ in eq. (5). In that same context, a good example is the definition of escort functions as $\phi_{k}\left(x_{k}\right)=x_{k}$, where the grow incentive is proportional to the proportion itself. In such case, ERD becomes the well-known replicator dynamics where the standard simplex,

$$
\Delta^{K}=\left\{\mathbf{x} \in \mathbb{R}^{K}: x_{k} \geq 0, \sum_{k=1}^{K} x_{k}=1\right\} \subset \mathbb{H}_{m=1}^{K},
$$

is positively invariant because $\phi_{k}(0)=0$ and $\phi_{k}\left(x_{k}\right)>0$ for $x_{k}>0$. In other words, if $x_{k}>0$ for all $k$ at $t=0$, then states $\mathbf{x}$ for $t>0$ will always remain within the convex set $\Delta^{K}$.

If all individuals play a single strategy $k$, the portion corresponding to that single strategy will be $x_{k}=1$. Thus, pure strategies are represented by the canonical base of $\mathbb{R}^{K}$ which are the vertices of the simplex $\Delta^{K}$. In general, for increasing escort functions such that $\phi_{k}(0)=0$, the standard simplex is invariant under ERD.

\section{A. Rest points of the dynamics}

Rest points of the dynamics are the zeros of the vector field defined by eq. (11). Some of these will in fact depend on the definitions of the escort functions and the underlying game. For instance, if escort functions are defined as $\phi_{k}\left(x_{k}\right)=x_{k}$, then at least $K$ rest points will exist, i.e. one per pure strategy [15]. In a different scenario, if escort functions are defined as $\phi_{k}\left(x_{k}\right)=1$, ERD becomes the orthogonal projection dynamics. In such a case, pure strategy rest points will no longer exist [16].

In general, rest points of ERD are states $\hat{\mathbf{x}}_{r p}$ for which payoff functions $f_{k}\left(\hat{\mathbf{x}}_{r p}\right)$ are equal to a given constant $\varepsilon$, considering only shares $k \in s p\left(\phi\left(\hat{\mathbf{x}}_{r p}\right)\right)$. Function $s p(\cdot)$ refers to the support of the vector input, i.e., only population shares $k \in\{1,2, \cdots, K\}$ whose escort functions are $\phi_{k}\left(\hat{x}_{k}\right)>0$ are considered. Since the concerned payoff functions are all $f_{k}\left(\hat{\mathbf{x}}_{r p}\right)=\varepsilon$, the weighted average payoff is also equal to $\varepsilon$, and $f_{k}\left(\hat{\mathbf{x}}_{r p}\right)-\bar{f}_{\phi}\left(\hat{\mathbf{x}}_{r p}\right)=\varepsilon-\varepsilon=0$, leading to $\dot{x}_{k}=0$ in eq. (1).

In particular, a Nash Equilibrium (NE) $\hat{\mathbf{x}}_{n e}$ of the underlying game is a rest point for the escort replicator dynamics if escort functions are defined to allow it. To explain this, consider that a NE must hold the condition $\mathbf{x} \cdot \mathbf{f}\left(\hat{\mathbf{x}}_{n e}\right) \leq \hat{\mathbf{x}}_{n e} \cdot \mathbf{f}\left(\hat{\mathbf{x}}_{n e}\right)$ [17, Section 19.5][15]. This condition implies that at the NE, the payoff functions are equal to a constant $f_{k}\left(\hat{\mathbf{x}}_{n e}\right)=\delta$ for strategies $k$ that are actually played (i.e., strategies $\left.k \in s p\left(\hat{\mathbf{x}}_{n e}\right)\right)$, and $f_{k}\left(\hat{\mathbf{x}}_{n e}\right) \leq \delta$ for strategies $k$ that are not played. Thus, if the $\mathrm{NE}$ lies in the interior of the simplex, (i.e. all strategies are played), then $\bar{f}_{\phi}\left(\hat{\mathbf{x}}_{n e}\right)=\delta$ and $f_{k}\left(\hat{\mathbf{x}}_{n e}\right)-\bar{f}_{\phi}\left(\hat{\mathbf{x}}_{n e}\right)=\delta-\delta=0$, leading again to $\dot{x}_{k}=0$ in eq. (1).

\section{B. Evolutionarily Stable States}

Regardless of the nature of the payoff landscape that can either be defined or not by the euclidean gradient of a potential function, and taking into account the symmetry of the underlying game, an equilibrium state $\hat{\mathbf{x}}$ defined by an inequality condition of the form of

$$
(\hat{\mathbf{x}}-\mathbf{x}) \cdot \mathbf{f}(\mathbf{x})>0,
$$


is known as an Evolutionarily Stable State (ESS) [1]. For contrast, from subsection II-A a Nash Equilibrium (NE) is defined by $\left(\hat{\mathbf{x}}_{n e}-\mathbf{x}\right) \cdot \mathbf{f}\left(\hat{\mathbf{x}}_{n e}\right) \geq 0$, which differs to the ESS definition in the evaluation of the payoff vector at the equilibrium state instead of its vicinity. Let us consider a payoff landscape $\mathbf{f}(\mathbf{x})$ defined by the euclidean gradient of a potential function $F(\mathbf{x})$, i.e. $\mathbf{f}(\mathbf{x})=\nabla F(\mathbf{x})$, and think of an unstable equilibrium (e.g., a saddle point). In such case, the payoff vector evaluated at the saddle point is zero valued which results in a valid NE condition, i.e., $\left(\hat{\mathbf{x}}_{n e}-\mathbf{x}\right) \cdot \mathbf{f}\left(\hat{\mathbf{x}}_{n e}\right)=0$. However, the payoff vector evaluated at a vicinity of that unstable equilibrium, $\mathbf{f}(\mathbf{x})$, will always point outward $\hat{\mathbf{x}}_{n e}$, forming an obtuse angle with vector $\left(\hat{\mathbf{x}}_{n e}-\mathbf{x}\right)$. This results in an unvalid ESS condition, $\left(\hat{\mathbf{x}}_{n e}-\mathbf{x}\right) \cdot \mathbf{f}(\mathbf{x})<0$ for states at the vicinity of the saddle point. To summarize, the ESS is a more refined definition of stable equilibrium state than that of NE. In a symmetric game set-up, ESS is equivalent to Strict $N E((\hat{\mathbf{x}}-\mathbf{x}) \cdot \mathbf{f}(\hat{\mathbf{x}})>0)[18]$.

\section{ERD with Euclidean gradient as payoff landscape}

Finally, as proved in [1], for payoff landscapes $\mathbf{f}(\mathbf{x})$ defined by the euclidean gradient of a potential function, the ERD, defined by (1), behaves as a gradient flow. This means that the trajectories from any initial state $\mathbf{x}(0)$ are such that the maximal direction of change is followed, and eventually a local optimum of the potential function is reached. This is a generalization of the Shahshahani gradient in replicator dynamics, which can be obtained with escort functions $\phi_{k}\left(x_{k}\right)=x_{k}$ [17, Section 19.5][19].

\section{TWO SIMPLEXES AND THEIR INTERSECTION}

For non-decreasing escort functions, and strictly positive on $0<x_{k}<1$, the author of [1] provides Lyapunov functions for ERD, proving the stability of an ESS. These Lyapunov functions depend on the escort functions definition [1], [20].

In this paper, escort functions are considered an asset and their characteristics are exploited to keep $\mathrm{x}$ in a constrained simplex $\Psi^{K}$ for all $t>0$. The new manifold, as the simplex, is $(K-1)$-dimensional, is also embedded in $\mathbb{R}^{K}$, and is a subspace of the hyper-plane $\mathbb{H}_{m}^{K}$. However, by intuitively exploiting the definition of the escort functions, $\Psi^{K}$ confines the state vector inside the intersection of two simplexes that represent upper and lower constraints on the state variables.

From a population dynamics perspective, this can be interpreted for instance as a natural limit on the use of a given strategy of the underlying game. In fact, these new definitions are proposed to develop an application of ERD in optimal distributed resource allocation scenarios, where these limited access to resources are a common issue. As it is demonstrated in this paper, ERD equipped with the proposed definitions is still able to reach a local ESS (a local strict NE). Even if the proposed escort functions are neither strictly positive nor nondecreasing on the interval $0<x_{k}<1$, a Lyapunov function can still be found for an equilibrium $\hat{\mathbf{x}}$ in the constrained simplex $\Psi^{K}$.

The first part of this section summarizes the characteristics of the desirable feasible set $\Psi^{K}$. The second part defines of the escort functions required to confine the vector state inside $\Psi^{K}$, and the reason behind these definitions.

\section{A. Desirable feasible region}

The desirable feasible region is defined as

$$
\Psi^{K}=\left\{\mathbf{x} \in \mathbb{R}^{K}: x_{k}^{l o} \leq x_{k} \leq x_{k}^{u p}, \sum_{k=1}^{K} x_{k}=m\right\},
$$

with $\Psi^{K} \subset \mathbb{H}_{m}^{K}$ and where $x_{k}^{l o}<x_{k}^{u p}$ for all $k$, and both can be larger or smaller than 1 in an engineering application context. If a population dynamics context is being represented by ERD, then $m=1$, and these boundaries should be restricted to $0 \leq x_{k}^{l o}<x_{k}^{u p} \leq 1$ for conceptual consistency (population shares must be non-negative). However, generally speaking in an engineering application context, these boundaries are not required to be positive, and $m$ is not required to be 1 .

Region $\Psi^{K}$ can also be defined as the intersection $\Psi^{K}=$ $\Delta_{u p}^{K} \cap \Delta_{l o}^{K}$, of simplexes $\Delta_{u p}^{K}$ and $\Delta_{l o}^{K}$, which can be expressed as,

$$
\begin{aligned}
\Delta_{u p}^{K} & =\left\{\mathbf{x} \in \mathbb{R}^{K}: x_{k} \leq x_{k}^{u p}, \sum_{k=1}^{K} x_{k}=m\right\} \subset \mathbb{H}_{m}^{K}, \\
\Delta_{l o}^{K} & =\left\{\mathbf{x} \in \mathbb{R}^{K}: x_{k} \geq x_{k}^{l o}, \sum_{k=1}^{K} x_{k}=m\right\} \subset \mathbb{H}_{m}^{K} .
\end{aligned}
$$

The set $\Psi^{K}$, simplexes $\Delta_{u p}^{K}$ and $\Delta_{l o}^{K}$, and even the standard simplex $\Delta^{K}$, are all convex polytopes that can be fully described by their set of vertices. For instance, the set of vertices for the standard simplex is the $K$-dimensional identity matrix $\mathbf{I}$ (i.e. the standard basis of $\mathbb{R}^{K}$ ). Similarly, the sets of vertices for simplexes $\Delta_{u p}^{K}$ and $\Delta_{l o}^{K}$ can be represented as the column vectors of matrices $\mathbf{C}_{u p}$ and $\mathbf{C}_{l o}$ respectively. These matrices are $K$ dimensional, and represent basis for $\mathbb{R}^{K}$ as well. Furthermore, the set of vertices of the intersection region can be packed as well in a matrix $\mathbf{C}_{\Psi}$ whose dimensions are $(K \times L)$ being $L$ the number of vertices of the intersection.

If these matrices are known, it is possible to redefine (7) and (8) equivalently as convex hulls as,

$$
\begin{aligned}
\Psi^{K} & =\left\{\mathbf{x}=\mathbf{C}_{\Psi} \eta: \eta \in \mathbb{R}^{L}, \eta_{l} \geq 0, \sum_{l=1}^{L} \eta_{l}=1\right\} \\
\Delta_{u p}^{K} & =\left\{\mathbf{x}=\mathbf{C}_{u p} \alpha: \alpha \in \mathbb{R}^{K}, \alpha_{k} \geq 0, \sum_{k=1}^{K} \alpha_{k}=1\right\} \\
\Delta_{l o}^{K} & =\left\{\mathbf{x}=\mathbf{C}_{l o} \beta: \beta \in \mathbb{R}^{K}, \beta_{k} \geq 0, \sum_{k=1}^{K} \beta_{k}=1\right\}
\end{aligned}
$$

Notice that sums $\sum_{l}^{L} \eta_{l}=1, \sum_{k}^{K} \alpha_{k}=1$, and $\sum_{k}^{K} \beta_{k}=1$ in eqs. 9), (10) and (11) are equal to 1, as opposed to sums $\sum_{k}^{K} x_{k}=m$ in eqs. (7) and (8). This is because eqs. (9), (10) (11) are convex hull representations, i.e., convex linear combinations of the vertices of the convex polytopes.

Matrices $\mathbf{C}_{u p}$ and $\mathbf{C}_{l o}$ can be easily found as it will be shown in subsection III-B However, for $\mathbf{C}_{\Psi}$ the number of vertices $L$ can be much larger that $K$ and enumerating all of them is a combinatorial problem, hard to solve computationally [21]. In [8], a similar scenario is considered but it is assumed that constraints are homogeneous for all the pure strategies, i.e., $x_{k}^{l o}=0$, and $x_{k}^{u p}=x^{u p}$ for all $k$. In that manuscript, vertices for eq. (9) are defined as mixed strategies of the underlying game, and states are driven towards an ESS by using the mixed strategist dynamics version of replicator dynamics [17, Section 19.6]. It is shown that the population can be divided in $\gamma$ pure strategies at most, where $\gamma$ is an integer that depends on $x^{u p}$ and lies somewhere between 1 and $K$ (i.e., $\gamma=\left\lfloor 1 / x^{u p}\right\rfloor$, where $\lfloor\cdot\rfloor$ is the floor operator). In that homogeneous scenario, the amount 
of vertices of $\Psi^{K}$ is relatively small for $\gamma$ close to 1 or close to $K$. However, even in this simplified scenario, the number of vertices becomes relatively large if $\gamma$ is near $K / 2$. For the general non-homogeneous case, finding the vertices of $\Psi^{K}$ is not a viable procedure [21].

\section{B. Convex hull matrices for the intersecting simplexes}

At the end of this subsection it will become clear that finding these matrices is not mandatory. However, the procedure is useful to illustrate the origin of the escort functions proposed in this paper. Let $\mathbf{x}^{u p}=\left[x_{1}^{u p}, x_{2}^{u p}, \cdots, x_{K}^{u p}\right]^{T}$ and $\mathbf{x}^{l o}=\left[x_{1}^{l o}, x_{2}^{l o}, \cdots, x_{K}^{l o}\right]^{T}$ be the column vectors containing the constraints that define simplexes $\Delta_{u p}^{K}$ and $\Delta_{l o}^{K}$ respectively. Matrices $\mathbf{C}_{u p}$ and $\mathbf{C}_{l o}$ are defined by

$$
\mathbf{C}_{u p}=\mathbf{x}_{u p} \mathbf{1}^{T}+\sigma_{u p} \mathbf{I}, \quad \mathbf{C}_{l o}=\mathbf{x}_{l o} \mathbf{1}^{T}+\sigma_{l o} \mathbf{I},
$$

where 1 is a $K$-dimensional column vector with all its entries equal to 1 . Scalars $\sigma_{l o}$, and $\sigma_{u p}$ are

$$
\sigma_{u p}=m-\sum_{k=1}^{K} x_{k}^{u p}, \quad \sigma_{l o}=m-\sum_{k=1}^{K} x_{k}^{l o},
$$

with $m=1$ if states lie in the hyper-plane $\mathbb{H}_{m=1}^{K}$ and upper and lower simplexes are subsets of that same hyper-plane. It is important to notice that $\sigma_{u p}$ and $\sigma_{l o}$ in eq. (13) must satisfy the conditions given by,

$$
\sigma_{u p}<0, \quad \sigma_{l o}>0 .
$$

Otherwise, the hyper-plane $\mathbb{H}_{m}^{K}$ (i.e., $\sum_{k=1}^{K} x_{k}=m$ ) would be unfeasible since constraints $x_{k}^{u p}$ would be too low (i.e. $\sum_{k=1}^{K} x_{k}^{u p}<m$ ), or constraints $x_{k}^{l o}$ would be too high (i.e. $\left.\sum_{k=1}^{K} x_{k}^{l o}>m\right)$.

Fig. 1 (a) illustrates the standard simplex $\Delta^{3}$ in $\mathbb{R}^{3}$ and a simplex of upper constraints $\Delta_{u p}^{3}$. As it was described, the column vectors of $\mathbf{C}_{u p}$ are the vertices of that simplex $\Delta_{u p}^{3}$ found by applying eq. (12). This simplex looks like it is inverted w.r.t. the standard simplex, which occurs because of the nature of its inequalities. Fig. $11(b)$ shows a simplex of lower constraints $\Delta_{l o}^{3}$. Again, the vertices of $\Delta_{l o}^{3}$ are found using eq. (12). Fig. 1 $(c)$ shows the intersection $\Psi^{3}=\Delta_{u p}^{3} \cap \Delta_{l o}^{3}$ of both simplexes. This diagram is useful to highlight that the number of vertices of the intersection depends on the constraints and maybe unpredictable for dimensions larger than $K=3$.

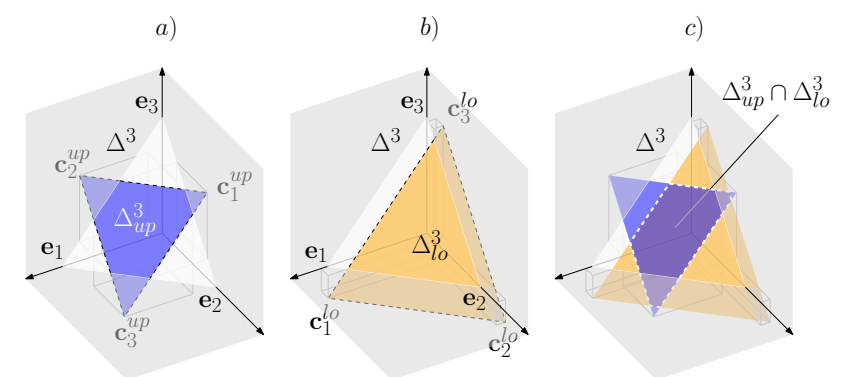

Fig. 1: In $\mathbb{R}^{3}:(a)$ standard simplex $\Delta^{3}$, and a simplex of upper constraints $\Delta_{u p}^{3} ;(b)$ standard simplex $\Delta^{3}$, and simplex of lower constraints $\Delta_{l o}^{3} ;(c)$ Intersection of both simplexes $\Delta_{u p}^{3} \cap \Delta_{l o}^{3}$.

\section{Definition of the proposed escort functions}

Let us consider a vector state $\mathbf{x} \in\left(\Delta_{u p}^{K} \cap \Delta_{l o}^{K}\right)$. From eqs. (10), (11) and (12) it is direct to verify that,

$$
\begin{gathered}
\alpha=\mathbf{C}_{u p}^{-1} \mathbf{x}=\frac{1}{\sigma_{u p}}\left(\mathbf{I}-\mathbf{x}_{u p} \mathbf{1}^{T}\right) \mathbf{x}=\frac{1}{\sigma_{u p}}\left(\mathbf{x}-\mathbf{x}_{u p} \mathbf{1}^{T} \mathbf{x}\right), \\
\beta=\mathbf{C}_{l o}^{-1} \mathbf{x}=\frac{1}{\sigma_{l o}}\left(\mathbf{I}-\mathbf{x}_{l o} \mathbf{1}^{T}\right) \mathbf{x}=\frac{1}{\sigma_{l o}}\left(\mathbf{x}-\mathbf{x}_{l o} \mathbf{1}^{T} \mathbf{x}\right),
\end{gathered}
$$

which are the representations of $\mathbf{x}$ in the basis $\mathbf{C}_{u p}$ and $\mathbf{C}_{l o}$ of $\mathbf{R}^{K}$. Since $\mathbf{1}^{T} \mathbf{x}=\sum_{k=1}^{K} x_{k}=m$, the entries of vectors $\beta$ and $\alpha$ are given by,

$$
\begin{aligned}
& \alpha_{k}=\frac{1}{\sigma_{u p}}\left(x_{k}-x_{k}^{u p}\right), \\
& \beta_{k}=\frac{1}{\sigma_{l o}}\left(x_{k}-x_{k}^{l o}\right) .
\end{aligned}
$$

Expressions (15) and 16 provide direct useful information of $\mathbf{x}$ approaching to the boundaries of $\Psi^{K}=\Delta_{u p}^{K} \cap \Delta_{l o}^{K}$. It is important to notice that $\alpha_{k}$ and $\beta_{k}$ depend only on $x_{k}$. Moreover, it must be stressed that taking the inverse of $\mathbf{C}_{u p}$ and $\mathbf{C}_{l o}$, or even computing them, is not a requirement for ERD.

As general observation, from a population dynamics perspective, the column vectors of $\mathbf{C}_{u p}$ and $\mathbf{C}_{l o}$, are the vertices of simplexes $\Delta_{u p}^{K}$ and $\Delta_{l o}^{K}$ respectively, and can intuitively be interpreted as the pure strategies in those simplexes. The portion of population using the $k$-th pure strategy in $\Delta_{u p}^{K}$ (or in $\Delta_{l o}^{K}$ ) depends only on the portion of population using the $k$-th pure strategy in $\Delta^{K}$.

Expressions 15$]$ and 16 are the first candidates for escort functions proposed in this paper. Some typical plots for $\phi_{k}\left(x_{k}\right)=\alpha_{k}=\left(x_{k}-x_{k}^{u p}\right) / \sigma_{u p}$ and $\phi_{k}\left(x_{k}\right)=\beta_{k}=$ $\left(x_{k}-x_{k}^{l o}\right) / \sigma_{l o}$ are shown in Fig 2 It is important to highlight
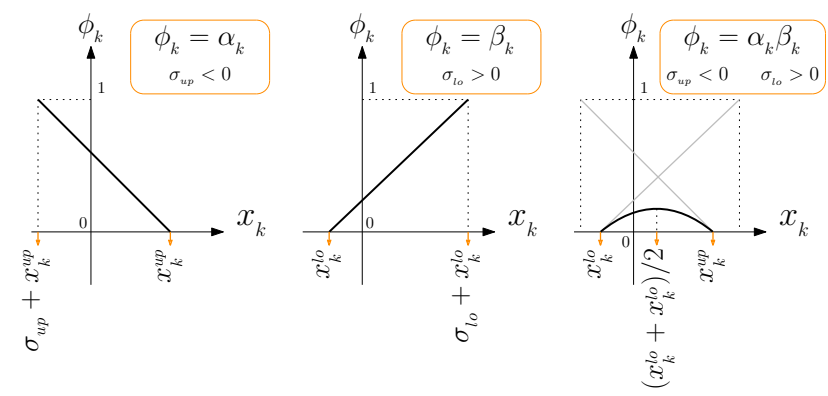

Fig. 2: Typical plots for the proposed escort functions $\left(\alpha_{k}, \beta_{k}\right.$, and $\alpha_{k} \beta_{k}$ ).

that $\alpha_{k}$ and $\beta_{k}$ reach the unity at $x_{k}=\sigma_{u p}+x_{k}^{u p}$ and $x_{k}=\sigma_{l o}+x_{k}^{l o}$ respectively. Additionally, $\alpha_{k}$ is monotonically decreasing while $\beta_{k}$ is monotonically increasing. This occurs because of the inequality conditions of eqs. (14). Moreover, the point where $\alpha_{k}$ or $\beta_{k}$ reach the unity, also corresponds to the point where the vertex of the corresponding simplex is placed. The third escort function given by $\phi_{k}\left(x_{k}\right)=\alpha_{k} \beta_{k}$ is the most interesting and relevant. It is quadratic, concave, positive at $x_{k}^{l o}<x_{k}<x_{k}^{u p}$ and it includes the desirable characteristics of the other two candidates. It is displayed at the right of Fig 2

\section{Using $\alpha_{k}, \beta_{k}$ and $\alpha_{k} \beta_{k}$ as escort functions}

As it was explained before, ERD guarantees $\mathbf{x} \in \mathbb{H}_{m}^{K}$ for all $t>0$. Given a continuous escort function $\phi_{k}\left(x_{k}\right)$, if at $t=0$, $x_{k}$ is such that $\phi_{k}\left(x_{k}\right)>0$ for all $k$, then the escort replicator dynamics will provide states $\mathbf{x}$ for $t>0$ such that $\phi_{k}\left(x_{k}\right) \geq 0$ 
for all $k$. From eq. (1), if $\phi_{k}\left(x_{k}\right)$ approaches to zero then $\dot{x}_{k}$ approaches to zero as well. Thus the zeros of the escort functions represent barriers for the feasible states $\mathbf{x}$, as it was explained for the replicator dynamics in section II]

If $\phi_{k}\left(x_{k}\right)=\alpha_{k}$, and at $t=0, x_{k}$ is such that $\alpha_{k}>0$ for all $k$, then the escort replicator dynamics will provide states $\mathbf{x}$ such that $\alpha_{k} \geq 0$. Since it is guaranteed that $\mathbf{x} \in \mathbb{H}_{m}^{K}$, from eq. (15) it is also guaranteed that,

$$
\Phi(\mathbf{x})=\sum_{k=1}^{K} \alpha_{k}=\frac{1}{\sigma_{u p}} \sum_{k=1}^{K}\left(x_{k}-x_{k}^{u p}\right)=1 .
$$

Thus, $0 \leq \alpha_{k} \leq 1$, or equivalently $\sigma_{u p}+x_{k}^{u p} \leq x_{k} \leq x_{k}^{u p}$ for all strategy $k$, and for all $t>0$. This implies that an escort replicator dynamics of the form:

$$
\dot{x}_{k}=\alpha_{k}\left(f_{k}(\mathbf{x})-\bar{f}_{\alpha}(\mathbf{x})\right)=\frac{\left(x_{k}-x_{k}^{u p}\right)}{\sigma_{u p}}\left(f_{k}(\mathbf{x})-\bar{f}_{\alpha}(\mathbf{x})\right),
$$

with eq. (2) as definition of the weighted average payoff, will always result in states $\mathbf{x} \in \Delta_{u p}^{K}$, i.e. the simplex $\Delta_{u p}^{K}$ is invariant under ERD of the form of eq. (17).

A similar reasoning applies if an escort function of the form $\phi_{k}\left(x_{k}\right)=\beta_{k}$ is employed. If at $t=0, x_{k}$ is such that $\beta_{k}>0$ for all strategy $k$, then $0 \leq \beta_{k} \leq 1$, or equivalently $x_{k}^{l o} \leq x_{k} \leq$ $\sigma_{l o}+x_{k}^{l o}$ for all strategy $k$, and for all $t>0$. Thus, an escort replicator dynamics of the form

$$
\dot{x}_{k}=\beta_{k}\left(f_{k}(\mathbf{x})-\bar{f}_{\beta}(\mathbf{x})\right)=\frac{1}{\sigma_{l o}}\left(x_{k}-x_{k}^{l o}\right)\left(f_{k}(\mathbf{x})-\bar{f}_{\beta}(\mathbf{x})\right),
$$

will provide states $\mathbf{x} \in \Delta_{l o}^{K}$, i.e. the simplex $\Delta_{l o}^{K}$ is invariant under ERD of the form of eq. (18).

Now let us evaluate the case where $\phi_{k}\left(x_{k}\right)=\alpha_{k} \beta_{k}$ is chosen as the escort function. If at $t=0, x_{k}$ is such that $\alpha_{k} \beta_{k}>$ 0 for all $k$, then it is also true that both $\alpha_{k}>0$ and $\beta_{k}>$ 0 at $t=0$. Again, given that the escort replicator dynamics guarantees $\mathbf{x} \in \mathbb{H}_{m}^{K}$ for $t>0$, then from eq. (15) and eq. [16, $\sum_{k=1}^{K} \alpha_{k}=1$ and $\sum_{k=1}^{K} \beta_{k}=1$ are also guaranteed for $t>0$. Then both intervals $0 \leq \alpha_{k} \leq 1$ and $0 \leq \beta_{k} \leq 1$ are respected, or equivalently both $\sigma_{u p}+x_{k}^{u p} \leq x_{k} \leq x_{k}^{u p}$ and $x_{k}^{l o} \leq x_{k} \leq$ $\sigma_{l o}+x_{k}^{l o}$ are respected. This last statement is only valid if the intersection exists. Thus, an ERD of the form

$$
\begin{aligned}
\dot{x}_{k} & =\alpha_{k} \beta_{k}\left(f_{k}(\mathbf{x})-\bar{f}_{\alpha \beta}(\mathbf{x})\right) \\
& =\frac{\left(x_{k}-x_{k}^{u p}\right)\left(x_{k}-x_{k}^{l o}\right)}{\sigma_{u p} \sigma_{l o}}\left(f_{k}(\mathbf{x})-\bar{f}_{\alpha \beta}(\mathbf{x})\right),
\end{aligned}
$$

will provide states $\mathbf{x} \in \Delta_{u p}^{K} \cap \Delta_{l o}^{K}$. Thus the intersection of the two simplexes is invariant under an escort replicator dynamics of the form of eq. (19). Taking into account the nature of the proposed escort functions, it is possible to propose candidates for Lyapunov functions to prove the stability of the dynamics at a local equilibrium point $\hat{\mathbf{x}}$.

\section{LOCAL STABILITY WITH ESCORT FUNCTIONS}

In this section, the main result of the paper is stated.

Theorem 1. Consider the generalized Escort Replicator Dynamics eq.1] with one the of the following escort functions $\phi_{k}\left(x_{k}\right)=\alpha_{k}, \phi_{k}\left(x_{k}\right)=\beta_{k}$, or $\phi_{k}\left(x_{k}\right)=\alpha_{k} \beta_{k}$, then an equilibrium state $\hat{\mathbf{x}}$ is locally stable.

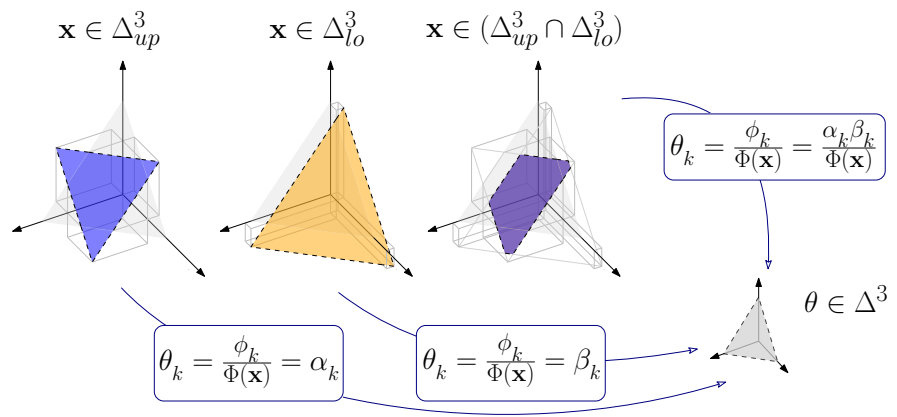

Fig. 3: Mapping of points $\mathrm{x}$ in the feasible regions $\Delta_{u p}^{K}, \Delta_{l o}^{K}$, and $\Delta_{u p}^{K} \cap \Delta_{l o}^{K}$, defined by the proposed escort functions $\left(\alpha_{k}\right.$, $\beta_{k}$, and $\alpha_{k} \beta_{k}$ respectively), to the standard probability simplex $\Delta^{K}$ where the distributions $\theta$ lie. Example simplexes for $K=3$, and some random constraints.

The proof is divided into two parts. First the escort functions $\phi_{k}\left(x_{k}\right)=\alpha_{k}$, and $\phi_{k}\left(x_{k}\right)=\beta_{k}$ are considered, then the third escort $\phi_{k}\left(x_{k}\right)=\alpha_{k} \beta_{k}$ is studied.

Proof. It has been observed that the escort functions $\phi_{k}\left(x_{k}\right)=$ $\alpha_{k}$ and $\phi_{k}\left(x_{k}\right)=\beta_{k}$ injectively map states $\mathbf{x}$ in $\Delta_{u p}^{K}$, and $\Delta_{l o}^{K}$ respectively, into states $\theta$ in the standard simplex $\Delta^{K}$, as it is shown on Fig. 3 This means that, through eq. (3), each state $\mathbf{x}$ in the simplexes $\Delta_{u p}^{K}$ or $\Delta_{l o}^{K}$, corresponds to only one image point $\theta$ in the standard simplex $\Delta^{K}$, where discrete probability distributions lie. It is then possible to consider a statistical distance measure function as candidate Lyapunov function, which is not novel for the replicator dynamics for instance [20], [18]. It is proposed to measure the statistical distance between two escort probability distributions: $\hat{\theta}$ corresponding to the equilibrium state $\hat{\mathbf{x}}$, and $\theta$ corresponding to a state $\mathbf{x}$ in the vicinity of $\hat{\mathbf{x}}$. The well-known Kullback-Leibler (K-L) divergence is a statistical distance measuring function, and for the distribution $\theta$ given by escort functions $\phi_{k}\left(x_{k}\right)=\alpha_{k}$, it takes the form,

$$
\begin{aligned}
D_{u p}^{\mathrm{K}-\mathrm{L}}(\hat{\theta}, \theta) & =\sum_{k \in s p(\hat{\theta})} \hat{\theta}_{k} \log \left(\frac{\hat{\theta}_{k}}{\theta_{k}}\right) \\
& =\sum_{k \in \operatorname{sp}(\phi(\hat{\mathbf{x}}))} \frac{\hat{x}_{k}-x_{k}^{u p}}{\sigma_{u p}} \log \left(\frac{\hat{x}_{k}-x_{k}^{u p}}{x_{k}-x_{k}^{u p}}\right),
\end{aligned}
$$

which is defined for all $k$ such that $\phi_{k}\left(\hat{x}_{k}\right)=\left(\hat{x}_{k}-x_{k}^{u p}\right) / \sigma_{u p}>$ 0 and $x_{k}<x_{k}^{u p}$. In other words, it is valid for states in the interior of the simplex $\Delta_{u p}^{K}$, or in its boundaries (which are also simplexes of reduced dimensions) if the ESS does not lie in the interior of $\Delta_{u p}^{K}$. Notice that the argument of the logarithm is positive since both terms of the division are negative. Similarly, the coefficient $\left(\hat{x}_{k}-x_{k}^{u p}\right) / \sigma_{u p}$ accompanying the logarithm is also positive because both terms in the division are negative by condition (14).

Intuitively, the K-L divergence is the weighted average of the logarithmic difference between the discrete probability distributions $\hat{\theta}$, and $\theta$, where the weighting coefficients are the probabilities $\hat{\theta}_{k}$. For $\phi_{k}=\alpha_{k}$, the K-L divergence on eq. 201 is a potential Lyapunov function candidate. 1) For the function 
$\phi_{k}=\alpha_{k}$ : Take the time derivative of the Lyapunov function candidate 20 and apply then chain rule to get,

$$
\dot{L}(\mathbf{x})=\sum_{k \in s p(\phi(\hat{\mathbf{x}}))}\left(\frac{-1}{\sigma_{u p}}\right) \frac{\hat{x}_{k}-x_{k}^{u p}}{x_{k}-x_{k}^{u p}} \dot{x}_{k}
$$

Replacing $\dot{x}_{k}$ by eq. (17), and reducing terms results in,

$$
\begin{aligned}
\dot{L}(\mathbf{x}) & =\frac{-1}{\sigma_{u p}^{2}} \sum_{k \in s p(\phi(\hat{\mathbf{x}}))}\left(\hat{x}_{k}-x_{k}^{u p}\right)\left(f_{k}(\mathbf{x})-\bar{f}_{\alpha}(\mathbf{x})\right) \\
& =\frac{-1}{\sigma_{u p}^{2}} \sum_{k \in s p(\phi(\hat{\mathbf{x}}))}\left(\hat{x}_{k}-x_{k}\right) f_{k}(\mathbf{x})=\frac{-(\hat{\mathbf{x}}-\mathbf{x}) \cdot \mathbf{f}(\mathbf{x})}{\sigma_{u p}^{2}},
\end{aligned}
$$

and if $\hat{\mathbf{x}}$ is an ESS, it fulfills the inequality condition (6). Thus, knowing that $\sigma_{u p}^{2}>0$, the expression before becomes $\dot{L}(\mathbf{x})=-\frac{1}{\sigma_{u p}^{2}}(\hat{\mathbf{x}}-\mathbf{x}) \cdot \mathbf{f}(\mathbf{x})<0$ only when $\mathbf{x} \neq \hat{\mathbf{x}}$. This implies that the chosen divergence is a valid Lyapunov function candidate if and only if $\hat{\mathbf{x}}$ is an ESS. Equivalently, since the chosen divergence is a valid Lyapunov function candidate, then $\hat{\mathbf{x}}$ must be an ESS.

2) For the function $\phi_{k}\left(x_{k}\right)=\beta_{k}$ : An identical procedure can be applied with with a similar Lyapunov function candidate for states in the interior of the simplex $\Delta_{l o}^{K}$. Due to space limitations, this exercise is left to the reader.

3) For the function $\phi_{k}\left(x_{k}\right)=\alpha_{k} \beta_{k}$ : Here, a divergence function can be constructed taking into account the procedures followed in [1] and [22] to construct generalized information divergences. First, a generalization of the natural logarithm, called the escort logarithm, is defined based on the proposed escort function as,

$$
\begin{aligned}
\log _{\phi}\left(x_{k}\right) & =\int_{\left(x_{k}^{l o}+x_{k}^{u p}\right) / 2}^{x_{k}} \frac{1}{\phi_{k}\left(x_{k}\right)} d x_{k} \\
& =\int_{\left(x_{k}^{l o}+x_{k}^{u p}\right) / 2}^{x_{k}} \frac{\sigma_{u p} \sigma_{l o}}{\left(x_{k}-x_{k}^{u p}\right)\left(x_{k}-x_{k}^{l o}\right)} d x_{k} \\
\log _{\phi}\left(x_{k}\right) & =\frac{\sigma_{u p} \sigma_{l o}}{x_{k}^{u p}-x_{k}^{l o}} \log \left(\frac{x_{k}^{u p}-x_{k}}{x_{k}-x_{k}^{l o}}\right) .
\end{aligned}
$$

The lower boundary of the integral is the argument that minimizes the $1 / \alpha_{k} \beta_{k}$ in the feasible interval $x_{k}^{l o} \leq x_{k} \leq x_{k}^{u p}$. It is also the argument that maximizes $\alpha_{k} \beta_{k}$ and the argument where the resulting escort logarithm is zero. Replacing (22) in,

$$
L(\mathbf{x})=\sum_{k \in s p(\phi(\hat{\mathbf{x}}))} \int_{x_{k}}^{\hat{x}_{k}}\left(\log _{\phi}(y)-\log _{\phi}\left(x_{k}\right)\right) d y,
$$

and analytically solving the integral results in,

$$
\begin{gathered}
L(\mathbf{x})=\sum_{k \in s p(\phi(\hat{\mathbf{x}}))} \frac{\sigma_{u p} \sigma_{l o}}{x_{k}^{u p}-x_{k}^{l o}}\left[\left(\hat{x}_{k}-x_{k}^{u p}\right) \log \left(\frac{\hat{x}_{k}-x_{k}^{u p}}{x_{k}-x_{k}^{u p}}\right)\right. \\
\left.-\left(\hat{x}_{k}-x_{k}^{l o}\right) \log \left(\frac{\hat{x}_{k}-x_{k}^{l o}}{x_{k}-x_{k}^{l o}}\right)\right]
\end{gathered}
$$

which is defined for all $k$ such that $\phi_{k}\left(\hat{x}_{k}\right)=\left(\hat{x}_{k}-x_{k}^{u p}\right)\left(\hat{x}_{k}-\right.$ $\left.x_{k}^{l o}\right) /\left(\sigma_{u p} \sigma_{l o}\right)>0$ (or equivalently $\left(\hat{x}_{k}-x_{k}^{u p}\right) / \sigma_{u p}>0$ and $\left(\hat{x}_{k}-x_{k}^{l o}\right) / \sigma_{l o}>0$ simultaneously), and $x_{k}^{l o}<x_{k}<x_{k}^{u p}$. In other words, it is valid only for states in the interior of the intersection $\Delta_{u p}^{K} \cap \Delta_{l o}^{K}$, or in its boundaries if the ESS does not lie in the interior of the intersection. The resulting escort divergence is strictly convex on intervals $x_{k}^{l o}<x_{k}<x_{k}^{u p}$, for all the strategies $k$, given an equilibrium state $\hat{\mathbf{x}}$ on the same interval. It is also $L(\hat{\mathbf{x}})=0$ and $L(\mathbf{x})>0$ for $\mathbf{x} \neq \hat{\mathbf{x}}$. It is possible to observe that the resulting divergence is a linear combination of both Kullback-Leibler divergences for the first two proposed escort functions, with coefficients depending on the allowed interval for each $x_{k}$. Taking the time derivative and then replacing $\dot{x}_{k}$ by 19 results in,

$$
\begin{aligned}
\dot{L}(\mathbf{x}) & =\sum_{k \in s p(\phi(\hat{\mathbf{x}}))} \frac{-\sigma_{u p} \sigma_{l o}\left(\hat{x}_{k}-x_{x}\right)}{\left(x_{k}-x_{k}^{u p}\right)\left(x_{k}-x_{k}^{l o}\right)} \dot{x}_{k} \\
& =-\sum_{k \in s p(\phi(\hat{\mathbf{x}}))}\left(\hat{x}_{k}-x_{k}\right) f_{k}(\mathbf{x})=-(\hat{\mathbf{x}}-\mathbf{x}) \cdot \mathbf{f}(\mathbf{x}) .
\end{aligned}
$$

Again, if $\hat{x}$ is an ESS it must fulfill condition 66. Then the last expression is,

$$
\frac{d L(\mathbf{x})}{d t}=-(\hat{\mathbf{x}}-\mathbf{x}) \cdot \mathbf{f}(\mathbf{x})<0,
$$

only when $\mathbf{x} \neq \hat{\mathbf{x}}$, which implies that the chosen divergence is a valid Lyapunov function candidate if and only if $\hat{x}$ is an ESS. It also means that if the chosen Lyapunov function candidate is valid, then $\hat{\mathbf{x}}$ has to be an ESS.

\section{DRIVING x TOWARDS A HYPER-PLANE $\mathbb{H}_{m}^{K}$}

The purpose of this section is to illustrate how the ERD can drive an initial state $\mathrm{x} \in \mathbb{H}_{m_{0}}^{K}$ defined by eq. (5), to an ESS that lies in a different hyper-plane $\mathbb{H}_{m}^{K}$, provided that the initial state fulfills certain conditions. Let us consider that the initial state $\mathbf{x}$ also lies in $\mathbb{B}^{K}=\left\{\mathbf{x} \in \mathbb{R}^{K}: x_{k}^{l o}<x_{k}<x_{k}^{u p}\right\}$, which is the interior of the hyper-rectangle defined by upper and lower boundaries. Recalling eq. (4), the hyper-plane where the state initially lies is invariant under the ERD. Thus, the dynamics asymptotically reach an ESS in both $\mathbb{H}_{m_{0}}^{K}$ and in the interior of $\mathbb{B}^{K}$.

However, since it is desirable to drive the initial state from $\mathbb{H}_{m_{0}}^{K}$ towards a local ESS in the hyper-plane $\mathbb{H}_{m}^{K}$, it is possible to use an augmented version $\mathbf{x}_{z}$ of the original state vector, defined by,

$$
\mathbf{x}_{z}=\left[\mathbf{x}^{T}, z\right]^{T}
$$

where $z$ is a slack state variable also described by ERD as,

$$
\dot{z}=\phi_{z}(z)\left(f_{z}(z)-\bar{f}_{\alpha \beta}\left(\mathbf{x}_{z}\right)\right) .
$$

The value of $z$ at $t=0$ is simply set to,

$$
z(0)=m-\sum_{k=1}^{K} x_{k}(0)=m-m_{0},
$$

and the payoff $f_{z}(z)$ for this slack portion of the population is defined such that $z$ is strongly attracted towards $-\zeta z(0)$, where $\zeta$ is a scalar such that $\zeta>1$. Please refer to section VI for an example of $f_{z}(z)$.

The escort function $\phi_{z}(z)$ is defined from upper and lower constraints $z^{l o} \leq z \leq z^{u p}$, such that $\phi_{z}(0)=0$. These limits are defined depending on the initial value $z(0)$. If $z(0)>0$, then $z^{l o}=0$ and $z^{u p}=z(0)+\varepsilon_{z}$. On the contrary, if $z(0)<0$, then $z^{u p}=0$ and $z^{l o}=z(0)-\varepsilon_{z}$. In both cases, a dummy constant $\varepsilon_{z}>0$ is used.

It can be noticed that the augmented state vector $\mathbf{x}_{z}$ lies in the hyper-plane $\mathbb{H}_{m}^{K+1}$ at $t=0$, and by ERD, it will always stay in that hyper-plane. However, since $z$ will be attracted towards $-\zeta z(0)$, it will be asymptotically blocked at $z=0$ 
by either $z_{u p}=0$ or $z_{l o}=0$. Thus, the original state vector will also asymptotically converge towards the hyper-plane $\mathbb{H}_{m}^{K}$. Moreover, the contribution of $f_{z}(\hat{z})$ to the weighted average payoff at a given rest point will be zero since at $\hat{z}=0$ its escort function is $\phi_{z}(0)=0$. Consequently the state $\mathbf{x}$ will be asymptotically driven to the original intersection $\Delta_{u p}^{K} \cap \Delta_{l o}^{K}$ and any rest point will have payoffs provided by the original payoff landscape.

\section{A. Strictly stable games and basin of attraction}

If the underlying symmetric game is strictly stable [16], also known as strictly contractive [23], it has a unique strict $\mathrm{NE} \hat{\mathbf{x}}$, or equivalently a globally ESS [23, Section 13.7.4]. In that context, the ERD defined by either eq. (17), (18) or (19), asymptotically converges to the ESS $\hat{x}$ from any initial state in the interior of the corresponding feasible region as shown in section IV.

The basin of attraction of $\hat{\mathbf{x}}$ for ERD in the context of a strictly stable symmetric game is the interior of the simplex $\Delta_{u p}^{K}$ if escort functions are $\phi_{k}\left(x_{k}\right)=\alpha_{k}$; the interior of the simplex $\Delta_{l o}^{K}$ if escort functions are $\phi_{k}\left(x_{k}\right)=\beta_{k}$; and the interior of the intersection $\Delta_{u p}^{K} \cap \Delta_{l o}^{K}$ if escort functions are $\phi_{k}\left(x_{k}\right)=\alpha_{k} \beta_{k}$.

Nevertheless, if a slack state variable $z$ is introduced as described in this section, then the basin of attraction for ERD for an underlying symmetric strictly stable game is defined according to the escort functions as:

- If escort functions are $\phi_{k}\left(x_{k}\right)=\alpha_{k}$, then the basin of attraction is defined by the hyper-octant $\mathbb{O}_{u p}^{K}=$ $\left\{\mathbf{x} \in \mathbb{R}^{K}: x_{k}<x_{k}^{u p}\right\}$

- If escort functions are $\phi_{k}\left(x_{k}\right)=\beta_{k}$, then the basin of attraction is defined by the hyper-octant $\mathbb{O}_{l o}^{K}=$ $\left\{\mathbf{x} \in \mathbb{R}^{K}: x_{k}>x_{k}^{l o}\right\}$

- Finally, if escort functions are $\phi_{k}\left(x_{k}\right)=\alpha_{k} \beta_{k}$, then the basin of attraction is defined by the hyper-rectangle $\mathbb{B}^{K}=$ $\left\{\mathbf{x} \in \mathbb{R}^{K}: x_{k}^{l o}<x_{k}<x_{k}^{u p}\right\}$

In the following section, some illustrative examples are introduced to clarify the purpose of the proposed escort functions.

\section{EXAMPLE WITH THE PROPOSED ESCORT FUNCTIONS}

In this section, an example in $\mathbb{R}^{4}$, using a symmetric game configuration with a non-potential function and unfeasible starting states is used to illustrate the use of the ERD with the proposed escort functions. Let us consider a payoff landscape defined by,

$$
\mathbf{f}(\mathbf{x})=\left[\begin{array}{ccc}
0 & -a & b \\
b & 0 & -a \\
-a & b & 0
\end{array}\right] \mathbf{x},
$$

where $a, b>0$. These payoff functions are usually employed to define an underlying game of the form of the classic Rock-Paper-Scissors game [18]. Now, let us describe a set of boundaries defining upper and lower simplexes $\Delta_{u p}^{3}=\left\{x_{1} \leq\right.$ $\left.0.75, x_{2} \leq 0.4, x_{3} \leq 0.5, x_{1}+x_{2}+x_{3}=0.95\right\}$, and $\Delta_{l o}^{3}=$ $\left\{x_{1} \geq 0.075, x_{2} \geq-0.05, x_{3} \geq 0.1, x_{1}+x_{2}+x_{3}=0.95\right\}$.

Let us assume that it is desirable to drive initial states to the hyper-plane $\mathbb{H}_{m=0.95}^{3}$. Initial states are assumed to fail to lie in the desired hyper-plane but they do lie in the interior of the hyper-rectangle $\mathbb{B}^{3}$. To drive states towards the hyper-plane $\mathbb{H}_{m=0.95}^{3}$, the strategy of section $\mathrm{V}$ is applied here. A slack variable $z$ is defined with payoff function given by $f(z)=$ $-2(z-\zeta z(0))$ with $\zeta=-5$ in this example. Moreover, for the underlying game and the desired hyper-plane $\mathbb{H}_{m=0.95}^{3}$, there is a global attractor at $\hat{\mathbf{x}}=[0.95 / 3,0.95 / 3,0.95 / 3]^{\mathrm{T}}$ for $a<b$ (in this example $a=1$, and $b=2$ ).

Fig. 4 (top) shows the hyper-rectangle $\mathbb{B}^{3}$ of the example, containing the intersection of simplexes $\Delta_{u p}^{3}$ and $\Delta_{l o}^{3}$. It also shows 4 trajectories of ERD starting at 4 different corners of the hyper-rectangle $\mathbb{B}^{3}$, and asymptotically converging to the global attractor $\hat{\mathbf{x}}$ located in the hyper-plane $\mathbb{H}_{m=0.95}^{3}$. Three of these trajectories start below the hyper-plane $\mathbb{H}_{m=0.95}^{3}$, and the fourth starts above the hyper-plane (upper left corner of the figure).
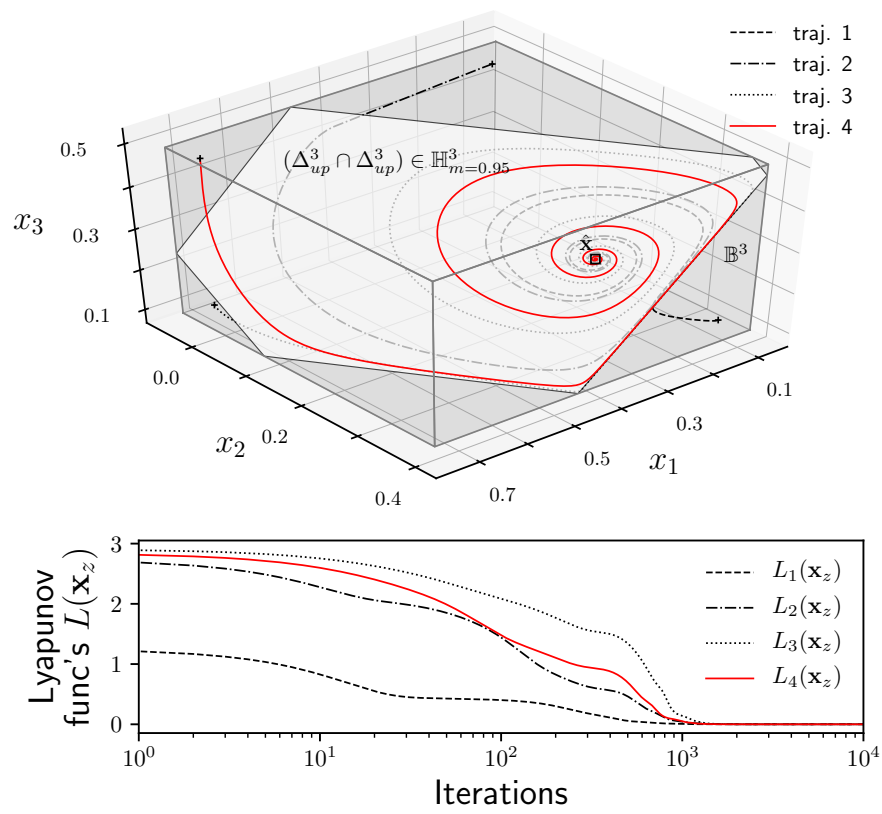

Fig. 4: (top) Four trajectories for example in section VI starting in the interior of the hyper-rectangle $\mathbb{B}^{3}$ defined by upper and lower constraints, and converging to the hyper-plane $\mathbb{H}^{3}$ where the global attractor $\hat{\mathbf{x}}$ lies. (bottom) Lyapunov functions of the form of eq. 23) evaluated for each of the four trajectories.

Lyapunov functions $L(\mathbf{x})$ like eq. 23) are computed for each trajectory, and can be observed in Fig. 4 (bottom). Let us notice the monotonic nature of the Lyapunov functions evaluated on each trajectory, and the convergence to zero corresponding to the convergence of the state vector towards the global attractor.

One of these trajectories starts at the hyper-plane $\mathbb{H}_{m=1.1643}^{3}$. For this case, $z(0)=0.95-1.1643=-0.2143$ and a dummy constant $\varepsilon_{z}=0.05$ is used to set to define its lower limit to $z^{l o}=-0.2643$. From [13), $\sigma_{l o}=1.0893$ and $\sigma_{u p}=-0.7$, including limits for $z$. For this case, Fig. $5(a)$ shows the state variables (including $z$ ) and their boundaries. It includes also the evolution of sums $\sum_{k}^{3} x_{k}+z$ and $\sum_{k}^{3} x_{k}$. It is possible to verify how $z$ starts at a negative value and converges to 0 , forcing the sum $\sum_{k}^{3} x_{k}$ to converge to $m=0.95$. Its is also important to notice that the sum $\sum_{k}^{3} x_{k}+z$ is always equal to $m=0.95$ for the whole trajectory even if some state variables $\left(x_{2}\right.$ and $z$ ) are initially negative. It is possible to observe that upper and lower boundaries are respected for each variable through the evolution. It is also possible to verify that the original state 


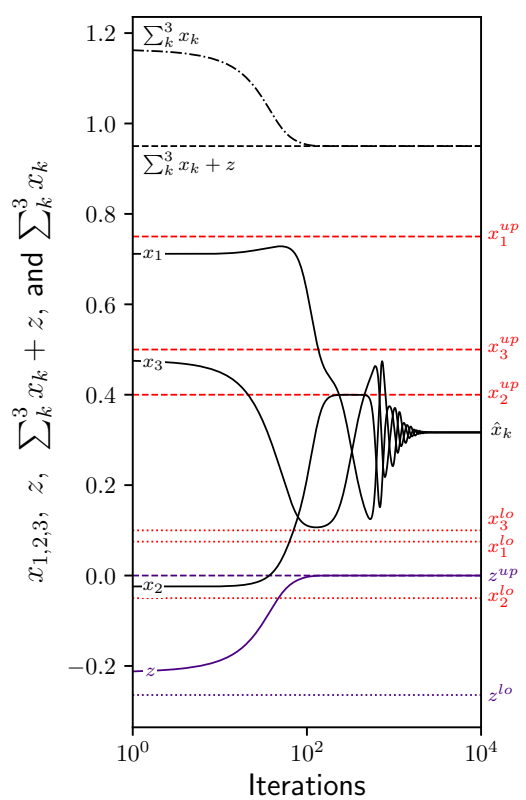

(a)
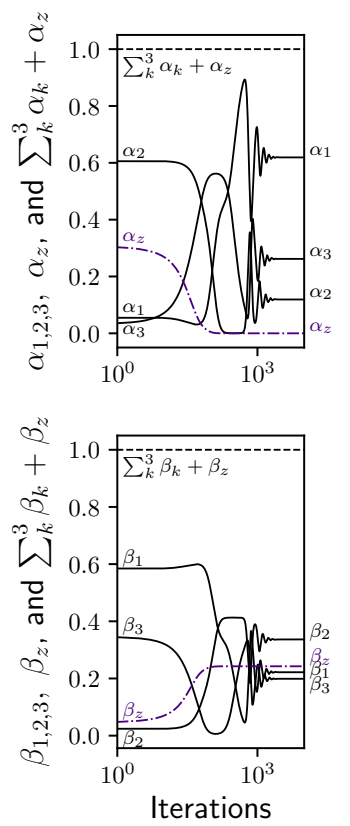

(b)
Fig. 5: A trajectory in the example of section $\mathrm{VI}$ (a) Upper and lower limits and evolution of state variables, and their sum. (b) Evolution of escort functions $\alpha_{1,2,3, z}, \beta_{1,2,3, z}$, and their sums.

variables converge to $\hat{x}_{k}=0.95 / 3$ which corresponds to the global attractor.

The charts in Fig. 5(b) show the escort functions $\alpha_{k}$ and $\beta_{k}$ for each of the state variables (including those of $z$ ). It is important to notice that the sums $\sum_{k}^{3} \alpha_{k}+\alpha_{z}$, and $\sum_{k}^{3} \beta_{k}+\beta_{z}$ are always equal to 1 for the whole trajectory (as it was for the sum of state variables and $z$ ). Moreover, let us notice that even if state variables are allowed to be negative, escort functions $\alpha_{k}$, $\beta_{k}$, and consequently their product $\alpha_{k} \beta_{k}$, are always positive for the whole trajectory.

\section{CONCLUSIONS}

In this paper, several details and features of ERD are presented, as well as the proposed novelty in the use of escort functions. In evolutionary game terms, the purpose of the proposed escort functions can be interpreted as upper and lower limits for the size of shares of the population that are allowed to play each of the pure strategies. This, for instance, can model some minimal quotas or limited hosting capacities of pure strategies. The employed analogies can be adapted to engineering problems of different nature where feasible regions can be, but are not required to be, defined by non-negative boundaries. The validity of the approach is tested with the local stability proofs of ERD under the application of the proposed escort functions.

As a future work, conditions for convergence of initial state that fail to initially lie in the interior of the hyper-rectangle formed by upper and lower constraints has to be studied. Special attention should be given to other types of escort functions that can model the intersection as well. For instance, escort functions can be defined such that for half of the feasibility interval a given escort function is employed and for the other half another escort function is used. In such cases, special attention should be given in the stability proof, considering the switching between escort maps. Moreover, modeling of non- orthogonal constraints should be considered in the definition of escort functions and the corresponding stability proofs.

\section{REFERENCES}

[1] M. Harper, "Escort evolutionary game theory," Physica D: Nonlinear Phenomena, vol. 240, no. 18, pp. 1411 - 1415, 2011.

[2] J. M. Smith, "Evolution and the theory of games," in Did Darwin Get It Right? Springer, 1988, pp. 202-215.

[3] J. M. Smith and G. R. Price, "The logic of animal conflict," Nature, vol. 246, no. 5427, pp. 15-18, 1973.

[4] W. H. Sandholm, "Evolutionary game theory," in Encyclopedia of Complexity and Systems Science. Springer, 2009, pp. 3176-3205.

[5] N. Quijano, C. Ocampo-Martinez, J. Barreiro-Gomez, G. Obando, A. Pantoja, and E. Mojica-Nava, "The role of population games and evolutionary dynamics in distributed control systems: The advantages of evolutionary game theory," IEEE Control Systems, vol. 37, no. 1, pp. 70-97, 2017.

[6] J. Barreiro-Gomez, N. Quijano, and C. Ocampo-Martinez, "Constrained distributed optimization: A population dynamics approach," Automatica, vol. 69, pp. 101-116, 2016.

[7] H. Tembine, E. Altman, R. El-Azouzi, and Y. Hayel, "Evolutionary games in wireless networks," IEEE Transactions on Systems, Man, and Cybernetics, Part B (Cybernetics), vol. 40, no. 3, pp. 634-646, 2010.

[8] A. Ovalle, J. Fernandez, A. Hably, and S. Bacha, "An electric vehicle load management application of the mixed strategist dynamics and the maximum entropy principle," IEEE Transactions on Industrial Electronics, vol. 63, no. 5, pp. 3060-3071, 2016.

[9] A. Ovalle, A. Hably, S. Bacha, G. Ramos, and J. Hossain, "Escort evolutionary game dynamics approach for integral load management of electric vehicle fleets," IEEE Transactions on Industrial Electronics, vol. 64, no. 2, pp. 1358-1369, 2017.

[10] A. A. Khan, M. Abolhasan, and W. Ni, "An evolutionary game theoretic approach for stable and optimized clustering in vanets," IEEE Transactions on Vehicular Technology, vol. 67, no. 5, pp. 4501-4513, 2018.

[11] A. A. A. Abass, L. Xiao, N. B. Mandayam, and Z. Gajic, "Evolutionary game theoretic analysis of advanced persistent threats against cloud storage," IEEE Access, vol. 5, pp. 8482-8491, 2017.

[12] K. Coninx, G. Deconinck, and T. Holvoet, "Who gets my flex? an evolutionary game theory analysis of flexibility market dynamics," Applied Energy, vol. 218, pp. 104-113, 2018.

[13] M. Harper and D. Fryer, "Lyapunov functions for time-scale dynamics on riemannian geometries of the simplex," Dynamic Games and Applications, vol. 5, no. 3, pp. 318-333, Sep 2015. [Online]. Available: https://doi.org/10.1007/s13235-014-0124-0

[14] P. Mertikopoulos and W. H. Sandholm, "Riemannian game dynamics," Journal of Economic Theory, vol. 177, pp. 315 - 364, 2018.

[15] J. Hofbauer and K. Sigmund, "Evolutionary game dynamics," Bulletin of the American Mathematical Society, vol. 40, no. 4, pp. 479-519, 2003.

[16] W. H. Sandholm, E. Dokumacı, and R. Lahkar, "The projection dynamic and the replicator dynamic," Games and Economic Behavior, vol. 64, no. 2, pp. $666-683,2008$, special Issue in Honor of Michael B. Maschler.

[17] J. Hofbauer and K. Sigmund, Evolutionary Games and Population Dynamics. Cambridge University Press, 1998.

[18] — The Theory of Evolution and Dynamical systems. Cambridge University Press, 1988.

[19] A. Menon, K. Mehrotra, C. K. Mohan, and S. Ranka, "Optimization using replicators," in Proceedings of the 6th International Conference on Genetic Algorithms, Pittsburgh, PA, USA, July 15-19, 1995, 1995, pp. 209-216.

[20] M. Harper, "Information geometry and evolutionary game theory," CoRR, vol. abs/0911.1383, 2009. [Online]. Available: http://arxiv.org/abs/0911. 1383

[21] D. Avis and K. Fukuda, "Reverse search for enumeration," Discrete Applied Mathematics, vol. 65, no. 1-3, pp. 21 - 46, 1996, first International Colloquium on Graphs and Optimization.

[22] J. Naudts, "Estimators, escort probabilities, and phi-exponential families in statistical physics," ArXiv Mathematical Physics e-prints, Feb. 2004.

[23] W. H. Sandholm, "Chapter 13 - population games and deterministic evolutionary dynamics," ser. Handbook of Game Theory with Economic Applications, H. P. Young and S. Zamir, Eds. Elsevier, 2015, vol. 4, pp. 703 - 778 . 